\title{
Incidental Finding of Isolated Rosai-Dorfman Disease in the Tonsils of a 4-Year-Old Girl: A Case Report and a Brief Review of Current Practice in Tonsillectomy Specimen Handling
}

\author{
Julianne Qualtieri ${ }^{1}$, Samir B. Kahwash ${ }^{2 *}$ \\ ${ }^{1}$ Department of Pathology, The Ohio State University, Columbus, USA; ${ }^{2}$ Department of Pathology and Laboratory Medicine, Na- \\ tionwide Children's Hospital, Columbus, USA. \\ Email: *Samir.Kahwash@NationwideChildrens.org
}

Received August $17^{\text {th }}, 2012$; revised September $17^{\text {th }}, 2012$; accepted October $4^{\text {th }}, 2012$

\begin{abstract}
We report herein a case of isolated Rosai-Dorfman disease (Sinus Histiocytosis with Massive Lymphadenopathy) diagnosed upon routine microscopic examination of tonsils in a child. The patient is a 4-year-old girl who had a history of sleep apnea, but no other significant medical history or other findings on physical examination. In this report, we discuss the various approaches to handling of tonsillectomy specimens. We also discuss the value of routine microscopic examination, providing a list of case examples of incidental, clinically important, findings that can be missed if a microscopic examination of tonsils was not performed. We finally discuss the clinical significance of finding histologic evidence of Rosai-Dorfman disease in a child.
\end{abstract}

Keywords: Tonsils; Rosai-Dorfman Disease; Microscopic Examination

\section{Introduction}

The value of routine submission of tonsillectomy tissue for microscopic examination is often brought into question, as a cost saving measure, given the low yield of clinically significant findings. Some advocate limiting pathologic examination to gross inspection, with submission of sections for microscopy reserved for tonsils that appear grossly abnormal or if the patient has a significant or unusual medical history [1]. Performing no examination at all of tissue has also been proposed by some authors [2]. While there is an obvious cost saving by not performing routine microscopic examination on tonsils in the above two approaches, this is only achieved at the risk of missing potentially important findings. A recent example is our case described below.

\section{Case Report}

The patient is a 4-year-old girl who presented for complaints of obstructive sleep apnea. No other significant medical history or physical findings were noted. In particular, there was no evidence of lymphadenopathy. She underwent tonsillectomy and adenoidectomy. Tonsils were

${ }^{*}$ Corresponding author. sent for pathologic examination, per protocol. At gross examination, no gross abnormalities were noted. Microscopic examination showed a conspicuous and discrete focus of large, histiocytes with abundant slightly eosinophilic cytoplasm, round nuclei and prominent nucleoli (Figures 1(a) and (b)). These histiocytic cells showed distinctive emperipolesis of lymphocytes, neutrophils, and plasma cells present (Figure 1(c)). An S100 immunohistochemical stain showed strong nuclear and cytoplasmic staining, confirming the diagnosis of Rosai-Dorfman disease (Figure 1(d)). Retrospective repeat gross examination of formalin fixed remainder of tonsilar tissue confirmed the absence of any other lesions or abnormalities. A review of the patient's records showed no evidence or reason to suspect involvement of other organs by Rosai-Dorfman changes, suggesting an isolated involvement of tonsils; an unusual presentation, with only rare cases reported in the literature [3].

\section{Discussion}

Microscopic examination of the vast majority of tonsils shows reactive non-specific follicular lymphoid hyperplasia, with or without active inflammation. A significant minority of specimens show gross aggregates of non- 

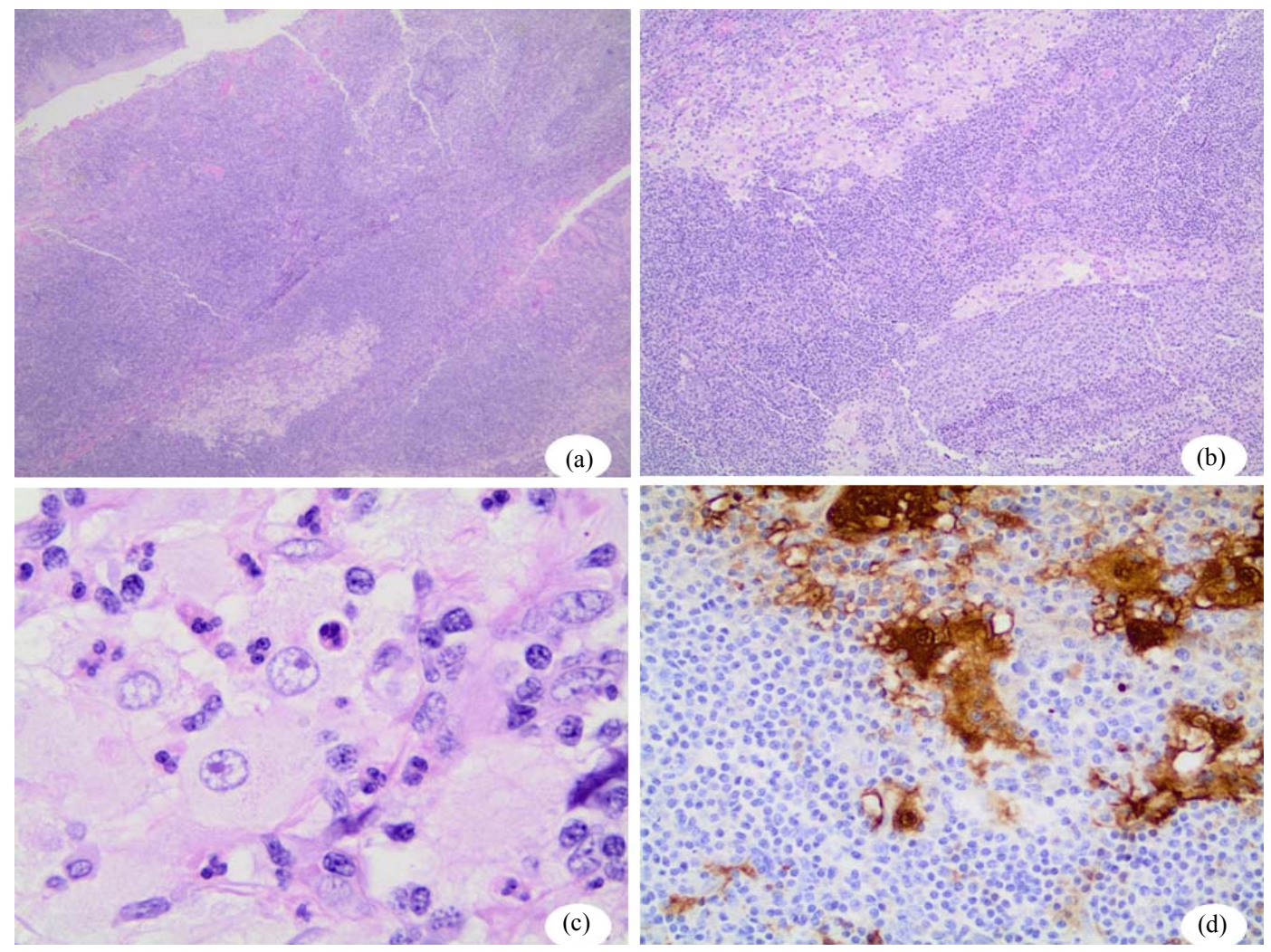

Figure 1. (a) and (b) H\&E stained sections of tonsil showing sheets of large histiocytic cells within and around lymphoid follicles; (c) At higher magnification, the typical appearance of Rosai-Dorfman cells is that of large histiocytic cells with abundant eosinophilic cytoplasm, phagocytozed lymphocytes or neutrophils, large round nuclei and prominent nucleoli; (d) Immunoperoxidase staining for $\mathrm{S} 100$ is positive in the cytoplasms and nuclei of lesional cells.

invasive actinomyces bacteria. Other pathologic findings are much less common, but clinically significant incidental findings are encountered.

A review of tonsillectomy/adenoidectomy specimen records at our institution over the last ten years demonstrated at least one case of each in the following clinically unexpected findings: Burkitt lymphoma, Myeloid Sarcoma, Rosai-Dorfman disease, Crohn's disease associated granulomatous inflammation, Tangier disease, toxoplasmosis, cat-scratch disease, granulomatous inflammation of unspecified etiology, acute Epstein-Barr virus (EBV) infection, and acute adenovirus infection.

Some of the above-mentioned findings may not be clinically significant, or represent self-limiting conditions, but others are critically important and time sensitive diagnoses (e.g. Burkitt Lymphoma and Myeloid Sarcoma) or represent significant findings that are too important to be missed (e.g. granulomatous inflammations). While unexpected clinically significant diagnoses are certainly rare, their impact on patient management may merit continuing routine examination.

Since most of the above-mentioned findings are not usually apparent grossly, the opportunity for making the diagnosis of rare disorders represents another advantage of routine microscopic examination. Additionally, routine submission of tonsilar tissue sections for microscopic examination will guarantee that a source of the patient's deoxyribonucleic acid (DNA) is available for possible testing, if needed. With the ever increasing importance of genetic testing and DNA analysis, the latter source may be of growing significance for some patients.

Finally, the paucity of clinical information submitted with surgical specimens further complicates the decision to design a protocol that limits submitting tissue for microscopic examinations based on meeting specified clinical indications or criteria. Regardless of the policy chosen, clear communication with clinicians regarding institutional criteria for tissue examination is essential for optimal patient care [4].

In our patient, the diagnosis of Rosai-Dorfman disease can potentially have implications and may necessitate clinical follow up. While most cases of Rosai-Dorfman's lymphadenopathy follow a self-limiting course with spontaneous resolution, an underlying immune dysfunction may be seen in a minority of patients. In addition, clinical follow up will be important as some patients may po- 
tentially show local relapses and/or involvement of vital organs [5].

\section{Acknowledgements}

The authors thank Maria Nunez, Administrative Assistant, for her help with the manuscript.

\section{REFERENCES}

[1] M. D. Williams and H. M. Brown, "The Adequacy of Gross Pathological Examination of Routine Tonsils and Adenoids in Patients 21 Years Old and Younger," Human Pathology, Vol. 34, No. 1, 2003, pp. 1053-1057. doi:10.1053/S0046-8177(03)00408-8

[2] S. P. Verma, T. Stoddard, I. Gonzalez-Gomez and J. A. Koempel, "Histologic Analysis of Pediatric Tonsil and Adenoid Specimens: Is It Really Necessary?" International Journal of Pediatric Otorhinolaryngology, Vol. 73,
No. 4, 2009, pp. 547-550.

doi:10.1016/j.ijporl.2008.11.001

[3] H. Z. M Amer, V. Prasad and S. B. Kahwash, "RosaiDorfman Disease of the Tonsil in a 4-Year-Old Boy: Case Report and Review of the Literature," International Journal of Pediatric Otorhinolaryngology, Vol. 6, No. 1, 2011, pp. 17-19. doi:10.1016/j.pedex.2009.12.003

[4] J. K. Chan and W. Y. Tsang, "Uncommon Syndromes of Reactive Lymphadenopathy," Seminars in Oncology, Vol. 20, No. 6, 1993, pp. 648-657.

[5] K. Sayed, H. Van Savell Jr., R. E. Hutchison, J. Kepner, M. P. Link, M. Schwenn, et al., "Review of Tonsillar Lymphoma in Pediatric Patients from the Pediatric Oncology Group: What Can Be Learned about Some Indications for Microscopic Examination?" Pediatric and Developmental Pathology, Vol. 8, No. 5, 2005, pp. 533-540. doi:10.1007/s10024-005-0043-6 